\title{
Dichotic temporal interactions: Fusion and temporal order
}

\author{
HARVEY BABKOFF \\ Bar-Ilan University, Ramat-Gan, Israel \\ and \\ Biometrics Research, New York State Department of Mental Hygiene \\ 722 West 168th Street, New York, New York 10032
}

\begin{abstract}
Several phenomena are associated with a monotonic increase in interaural temporal asymmetry $(\Delta t)$ of equally intense auditory stimuli: lateralization of a fused stimulus in the direction of the lead ear, when $\Delta \mathrm{t}$ is in the microsecond range, the subsequent breakup of the fused stimulus with $\Delta \mathrm{t}$ in the millisecond range, followed by temporal order judgments at $\Delta$ ts longer than 20 msec. Two of these binaural phenomena are investigated, using the same experimental paradigm: the breakup of the fused sound into two separately perceived stimuli and the judgment of temporal order. A V-shaped function describes the relationship of discrimination to interaural temporal asymmetry. The left segment of this function, decreasing as $\Delta \mathrm{t}$ increases from 2 to $8 \mathrm{msec}$, crosses the $75 \%$ discrimination level at a dichotic temporal separation of $6 \mathrm{msec}$ and reflects the breakup of the fused stimulus. The right segment of this function, increasing from 12 to $128 \mathrm{msec}$, crosses the $75 \%$ discrimination level at a dichotic temporal separation of $18 \mathrm{msec}$, reflecting temporal order discrimination. The data are discussed in terms of the literature on temporal order judgments, and considered in terms of an overall framework which assumes that all of the phenomena which occur as a function of increased $\Delta \mathrm{t}$ result from different processing operations on the temporal continuum separating the dichotic stimuli (Sternberg \& Knoll, 1973).
\end{abstract}

The judgment of the correct order of two successive stimuli (either identical or nonidentical), presented to the same sensory modality or to two different modalities has been investigated by many authors over the past 15 years (e.g., Babkoff \& Sutton, 1963, 1971; Carmon \& Nachshon, 1971; Efron, 1963, 1973; Hirsh, 1959; Hirsh \& Sherrick, 1961; Homick, Elfner, \& Bothe, 1969; Oatley, Robertson, \& Scanlan, 1969; Patterson \& Green, 1970; Robinson, 1967; Rutschmann, 1966, 1973; Rutschmann \& Link, 1964; Swisher \& Hirsh, 1972; Thor, 1967). Several general tindings have, by now, been verified by almost all of these authors. The function relating discrimination of temporal order to the temporal separation between the two stimuli is monotonic when the discrimination is not dependent upon some complex interaction between the two stimuli whose order is to be perceived. The "threshold" for the perception of temporal order, under these circumstances, has been reporied by Hirsh and Sherrick (1961) to be between 15 and $20 \mathrm{msec}$ for trained subjects regardless of the mode of stimulation. For untrained subjects, Efron (1963) reported threshold values of $60 \mathrm{msec}$.

This research was performed under Grant MH18422 from the National Institute of Mental Health, United States Public Health Service. The author wishes to acknowledge the aid of Robert Laupheimer and Raymond Simon for the design and maintenance of the equipment, and to thank Dr. M. Wallach, Director of Brooklyn State Hospital, for the use of Brooklyn State Hospital facilities. The author has also benefited from the advice and encouragement of Dr. S. Sutton.
Long-duration intramodal stimuli, differing only with respect to onset asynchrony, apparently require longer temporal separations (onset asynchronies) than shorter stimuli or stimuli differing with respect to offset asynchrony. (Compare results reported by Oatley, Robertson, \& Scanlan, 1969, to those reported by Efron, 1973).

A second general finding is that where there is complex interaction between intramodal stimuli, temporal order information can be utilized by that sensory system, although the temporal separation is much shorter than the threshold for temporal order judgment. When stimuli of different wavelength rapidly follow one another, this information may be encoded as color changes rather than order judgment (Efron, 1973). In addition, two spatially separated visual targets, stimulated asynchronously, may give rise to a judgment based on motion, rather than a judgment of temporal order, thus yielding what appears to be psychophysical temporal order thresholds of less than $10 \mathrm{msec}$ (Swisher \& Hirsh, 1972). When a pair of short-duration monaurally presented tone bursts of different frequencies $(\Delta \mathrm{f}=$ $200 \mathrm{~Hz}$ ) are presented with asynchronous offsets, subjects are able to discriminate them from a mirror-image pair with an offset asynchrony of 2.0 msec, since a different dominant pitch is associated with each of the two patterns (Efron, 1973). With longer duration stimuli, however, offset asynchronies of $20 \mathrm{msec}$ and longer are necessary for discrimination (Patterson \& Green, 1970; Efron, 
1973). Monaural temporal intervals of 1.5 to $6.0 \mathrm{msec}$ are sufficient for discrimination between mirrorimage patterns of two unequal intensity transients in which one pair consists of an intense stimulus followed by a less intense stimulus $(1.5 \leqslant \Delta t \leqslant 6.0)$ and the other pair consists of the mirror image (same $\Delta t$ ), although these intervals are not sufficient to judge temporal order of the individual members of the pair of stimuli (Babkoff \& Sutton, 1971).

The auditory system has been shown to be capable of resolving very small temporal separations between stimuli. especially when the mode of stimulation is binaural. For example, a pair of transients, asynmetrical with respect to time $(\Delta t)$ of the order of $20-40 \mu \mathrm{sec}$. is perceived as one stimulus displaced from the median plane in the direction of the ear receiving the earlier stimulus. If the interaural time asymmetry $(\Delta t)$ is increased to between 0.5 and $1 \mathrm{msec}$. most subjects report hearing a unitary stimulus located at the lead ear. When the interval between dichotic stimuli is increased to approximately 2-4 msec, two sounds are reported-one at the ear receiving the earlier stimulus (lead ear) and a soft, just perceptible sound at the ear receiving the second stimulus (lag ear) (Babkoff \& Sutton, 1963; Békésy, 1960; Rosenzweig \& Rosenblith, 1950).

Further increases in interaural time asymmetry lead to the ability to judge temporal order (Hirsh \& Sherrick, 1961; Trimble, 1928). One might have predicted, therefore, a monotonic function relating discrimination of dichotic temporal order to dichotic temporal interval (Hirsh \&Sherrick, 1961). However, since, at short dichotic intervals $(2-4 \mathrm{msec})$, the lead stimulus is reported as louder than the lag stimulus, subjects can utilize this loudness cue to judge temporal order (Babkoff \& Sutton, 1963). The perceived loudness difference between the lead and lag stimuli decays as a function of the time separating them (Babkoff \& Sutton, 1963). The ability to utilize this cue to discriminate temporal order also deteriorates as a function of $\Delta t$, leading to a decrease in discrimination as dichotic temporal interval increases.

Two trends should thus appear in a function relating discrimination of the dichotic temporal order of short-duration stimuli to the temporal interval separating them. At very shoft $\Delta$ ts, where an interaural loudness cue exists, discrimination should be at a high level. As $\Delta t$ increases, interaction between the dichotic stimuli decays, resulting in a decrease of loudness cue and of discrimination level. At longer $\Delta t s$, however, temporal order judgment should increase as a function of $\Delta t$.

In a previous publication (Babkoff \& Sutton, 1963), we presented data on the early part of such a function relating discrimination of temporal order to dichotic temporal intervals ranging from 2 to $12 \mathrm{msec}$. The purpose of this paper is to investigate discrimination of temporal order to dichotic temporal separations ranging over $1.8 \log$ units, and thus to obtain psychophysical data on the use of temporal interval information by the auditory system over a larger $\Delta t$ range.

\section{APPARATUS AND METHOD}

The design of the apparatus permitted independent control of the intensity and temporal relationship of the binaurally presented pulses, as well as the length of the intertrial intervals. The interval between the dichotic stimuli was varied from 2 to $128 \mathrm{msec}$. This interval was generated by a crystal-controlled timer (local design) calibrated and monitored with a Systron-Donner counter-timer. Model 1034. Error did not exceed $0.05 \%$. Intertrial intervals were 10 sec.

The apparatus is described in detail elsewhere (Babkoff. Sutton. \& Barris, 1973).

The subject. wearing a pair of Sharpe HL-10 circumaural earphones. was seated in a sound-treated booth. A panel in front of the subject had a warning light and two response keys. The subject was instructed to attend to the warning light and to the stimuli which were presented after the onset of the warning light. The subject was instructed that, it he heard two stimuli at the two ears, he was to indicate which stimulus occurred first by depressing the right key if the first stimulus appeared at the right ear, the left key if the first stimulus appeared at the left ear. If he heard one stimulus (possible at $\Delta t 62 \mathrm{msec}$ ), the subject was to depress the button corresponding to the ear at which the single stimulus appeared.

The design used in this study, requiring the subject to respond as to which ear received the first stimulus, is a variation of the two-alternative forced-choice methodology. This methodology does not specity whether the independent variable should be maintained constant within a block of trials or randomized from trial to trial. This seemingly minor variation raises rather subtle issues which cannot be dealt with here, nor have they, in fact, been resolved. It might be pointed out that keeping the independent variable constant within a block of trials makes it easier for the subject to identify the relevant cue, but by its very nature, permits the subject, arbitrarily, to select and become familiar with one cue, ignoring others (Kietzman \& Sutton, 1968). In this experiment, the independent variable $(\Delta t)$ was constant within a block of 20 trials, but was randomized from block to block, with the constraint that no $\Delta t$ be presented twice before the other $\Delta$ ts were presented once. The ear to which the first stimulus was to be presented was randomized from trial to trial.

The monaural threshold of each subject was measured before the experiment began by the method of limits and monitored throughout the experiment. As no large changes in threshold occurred, these estimates were maintained throughout. Stimulus intensity at each ear was set at $30 \mathrm{~dB}$ re monaural threshold. Four subjects participated in this experiment. Ten different conditions of dichotic interpulse interval $(\Delta t)$ were used. Each condition was presented 120 times to three of the four subjects (six blocks of 20 trials each) and 100 times (five blocks of 20 trials each) to the fourth subject. Thus, the data are based on 1,200 trials for each of three of the four subjects and 1,000 trials for the fourth subject.

\section{RESULTS}

The data are plotted for the four subjects separately in Figure 1 as percent discrimination on the ordinate as a function of dichotic temporal interval $(\Delta t)$ on the abscissa in logarithmic units. Although intersubject variability is seen in terms of the absolute discrimination levels, the trends of all subjects are similar; a high discrimination level at the short $\Delta t s$, followed by a decrease in discrimination in the mid- $\Delta t$ range, followed by a rise in discrimination at the 


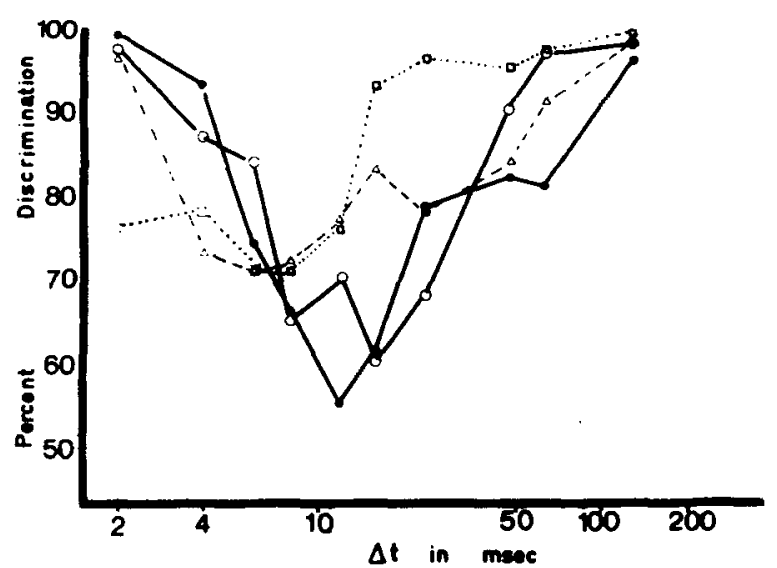

Figure 1. Percent discrimination is plotted on the ordinate as a function of the dichotic temporal interval separating the transient stimuli $(\Delta t)$ on the abscissa in logarithmic units. Data are shown separately for four subjects. See text for full explanation.

longer $\Delta$ ts. The data of the four subjects were analyzed by a one-way analysis of variance for repeated neasurements (Table 1 ). The main effect. dichotic temporal interval. $\Delta t$, is highly significant $(p \leqslant .001)$. The data of the four subjects were averaged and plotted in Figure 2 as percent discrimination as a function of dichotic temporal interval.

The function relating percent correct discrimination to dichotic temporal interval $(\Delta t)$ is nonmonotonic. consisting of two segments, a descending left limb, decreasing from $92 \%$ at $\Delta t=$ 2 misec to $68 \%$ at $\Delta t=8$, and an ascending right limb. ascending from $69 \%$ at $\Delta t=12 \mathrm{msec}$ to $98 \%$ at $\Delta t=128 \mathrm{msec}$. Note that the design allowed the subject to guess correctly. by chance, on $50 \%$ of the trials. i.e.. the expected minimum point of the function should be $50 \%$. It appears, in fact, that this function is constructed of two segments, one decreasing as a function of increases in $\Delta t$, the other increasing as a function of increases in $\Delta t$, with the two functions intercepting at approximately $\Delta t=$ $10 \mathrm{msec}$.

A second analysis was performed on these data, based on the assumption that two psychometric mechanisms of opposite sign are contributing to the shape of the function. The data were subdivided into two groups; $2 \mathrm{msec} \leqslant \Delta t \leqslant 8 \mathrm{msec}$, and $12 \mathrm{msec} \leqslant \Delta t$ $\leqslant 128 \mathrm{msec}$. Each group of data was analyzed by probit analysis (Finney, 1947), and by a least squares technique on an IBM 360/50 computer. The results are presented in Table 2 , which indicates that both functions can be fitted by the cumulative gaussian distribution ( $\chi^{2}$ not significant) when response frequency is plotted as a function of dichotic temporal separation $(\Delta t)$ in logarithmic units. The descending segment of the function crosses $75 \%$ correct discrimination at $\Delta t=6 \mathrm{msec}$, while the ascending segment of the function crosses $75 \%$ correct discrimination at $\Delta \mathrm{t}=18 \mathrm{misec}$. If these values are taken as threshold $(\mu)$ estimates and the $\sigma$ evaluated accordingly, then the coefficient of variation, $\sigma / \mu$. for the descending function is 1.06 , while for the ascending function it is 0.72 .

The least squares analysis indicates that discrimination level in probit scores plots linearly as a function of $\Delta t$ in log units for both functions ( $F=$ 942.43. $\mathrm{p} \leqslant .001$, for the descending function, and $\mathrm{F}$ $=124.73 . \mathrm{p} \leqslant .001$, for the ascending function) and accounts for $97 \%$ to $99 \%$ of the variance.

The probit and least squares analyses justify the treatment of the results as two separate psychometric functions intercepting at approximately $\Delta t=$ 10 msec.

\section{DISCUSSION}

The results indicate that temporal order discrimination is related to dichotic temporal asymmetry ( $\Delta t)$ by a nonmonotonic, V-shaped function beginning with a high level of discrimination at short $\Delta \mathrm{ts}$, decreasing as $\Delta \mathrm{t}$ increases to $8 \mathrm{msec}$, then increasing as $\Delta t$ increases further from 12 to $128 \mathrm{msec}$.

When the data are analyzed as if constructed of two psychometric functions, the left function crosses the $75 \%$ discrimination level at approximately $6 \mathrm{msec}$. while the right function crosses the $75 \%$ discrimination level at approximately $18 \mathrm{msec}$. These results are thus consistent with the hypothesis that interaction between members of a dichotic pulse pair. allowing the use of temporal order information. is a decreasing function of the temporal interval $(\Delta t)$ separating them. The left $\operatorname{limb}$ of the function represents the decay of dichotic interaction within the auditory nervous system and, thus, a modality-bound mechanism. The $\Delta t$ at $w$ hich the right function crosses the $75 \%$ discrimination level. $18 \mathrm{msec}$, is consistent with the threshold of temporal order judgment reported in the literature by Hirsh and Sherrick (1961), for inter-as well as intramodality stimuli. The right limb of the function represents a more central mechanism. nonmodality bound, which receives inputs from all modalities regarding temporal order. A larger coefficient of variation $(\sigma / \mu)$ for the descending function (1.06) than for the ascending

Table 1

Results of Analysis of Variance

\begin{tabular}{lcccc}
\hline Source & SS & df & MS & F \\
\hline $\begin{array}{l}\text { Dichotic Temporal } \\
\text { Interval }(\Delta \mathrm{t})\end{array}$ & 3756.50 & 9 & 417.389 & $4.7688^{*}$ \\
$\begin{array}{l}\text { Subjects Within } \\
\text { Groups }\end{array}$ & 290.875 & 3 & 96.958 & \\
$\begin{array}{l}\Delta t \text { by Subjects } \\
\text { Within Groups }\end{array}$ & 2363.188 & 27 & 87.525 & \\
Total & & 39 & & \\
\hline
\end{tabular}

${ }^{*} p \leqslant .001$ 


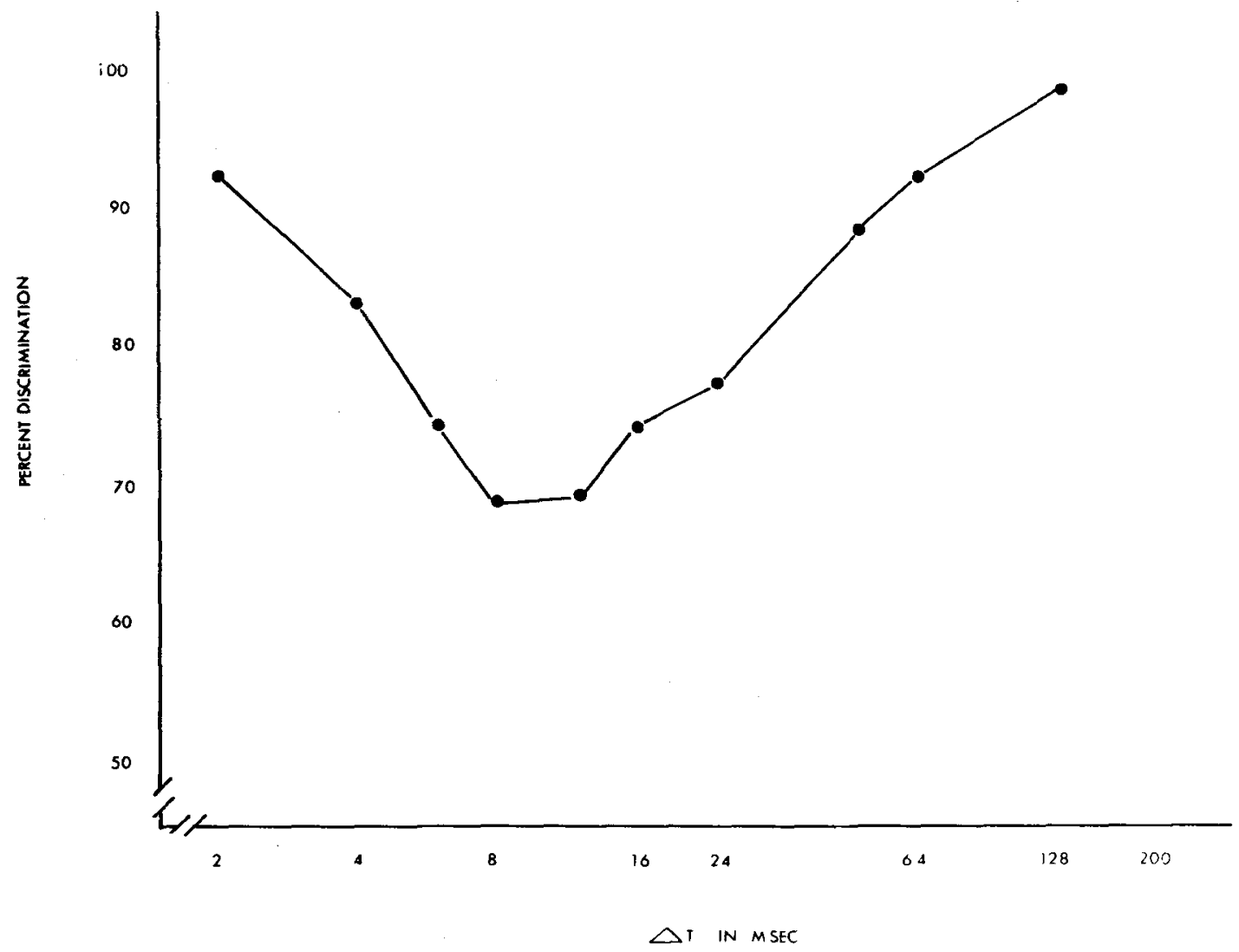

Figure 2. Percent discrimination is plotted on the ordinate as a function of the dichotic temporal interval separating the transient stimuli (At) on the abscissa in logarithmic anits. Average data for four subjects are shown. See text for full explanation.

function $(0.72)$ indicates a relatively larger amount of variance for the breakup of the fused image than for the temporal-order judgment. Perhaps the "fusednonfused" judgment is more restricted in the stimulus dimensions along which a judgment can be made than the more central order judgment which has more stimulus dimensions along which a decision as to order can be made. This may have led to a relative uncertainty of judgment for fusion or nonfusion leading to greater relative variability for this judgment.

Does an overall framework exist which can account for the data represented by both limbs of the temporal-discrimination/dichotic-interval function?

Sternberg and Knoll (1973) have recently presented a general model to explain temporal order judgments in which a "decision function" converts a difference in central "arrival time" of two sensory signals into an order judgment.

Sternberg and Knoll extend this general order-judgment model to include binaural lateralization phenomena by adding the provision that several decision functions may be interspersed along the way in a sensory system which receive and weigh arrival-time differences of inputs from two channels (in this case, from the two ears). Each channel performs processing operations on the signal prior to its arrival at a given decision function. The duration of the processing is inversely related to stimulus intensity. The arrival time difference at the $\mathrm{n}^{\text {th }}$ decision function is determined by the sum of the durations of the $n-1$ processes prior to it. This means that the arrival latency of the signal from that channel at the $n^{\text {th }}$ decision function is determined by the

Table 2

Result of Probit and Least-Squares Analyses for Temporal Order Discrimination as a Function of Dichotic Temporal Interval

\begin{tabular}{|c|c|c|c|c|c|c|}
\hline & $\begin{array}{c}\Delta \mathrm{t} \text { for } 75 \% \\
\text { Discrimination }\end{array}$ & $\sigma / \mu$ & $x^{2}$ & df & $\mathrm{F}$ & $\mathrm{R}^{2}$ \\
\hline $\begin{array}{l}\text { Descending Limb } \\
2 \mathrm{msec} \leqslant \Delta t \leqslant 8 \mathrm{msec} \\
\text { Ascending Limb } \\
12 \mathrm{msec} \leqslant \Delta t \leqslant 128 \mathrm{msec}\end{array}$ & $18 \mathrm{msec}$ & 1.06 & $\begin{array}{c}.0862 \\
\text { n.s. } \\
5.608 \\
\text { n.s. }\end{array}$ & (4) & $942.43^{*}$ & .97 \\
\hline
\end{tabular}


addition of the durations of all $\mathrm{n}-1$ processing operations prior to it.

When applying this model to binaural stimulation, one notes that the stimulus parameters which affect the lateralization of dichotic stimuli separated by very short temporal intervals (of the order of microseconds), such as frequency and interaural intensity asymmetry (Babkoff, Sutton, \& Barris, 1973; David, Guttman, \& van Bergeijk, 1959; Deatherage \& Hirsh, 1959; Green \& Henning, 1969; Harris, 1960), also affect the breakup of the fused image into two dichotic stimuli (Babkoff \& Sutton, 1966). This lends support to a general model in which all of these phenomena- occurring as a function of increased $\Delta t$, can be considered within the framework of different processing operations on the temporal continuum separating dichotic stimuli, beginning with the phenomena of "centering" $(\Delta t=0)$ and including temporal order judgment when $\Delta t>$ 20 msec.

The data presented in this paper, if understood in terms of the Sternberg and Knoll model, may represent the time constants of at least two decision functions, each operating on arrival latencies after a different number of processing operations. The left limb of the function (Figure 2) represents the decay of dichotic interaction at a lower level decision function related solely to auditory inputs. The right limb of the function represents the operation of a more central decision function which is concerned with temporal order judgments and which receives inputs from all sensory systems.

Because of the additivity aspect of the decision function operating on arrival time differences, the more processing required of the input before it arrives at the function. the greater will be the effect of intensity asymmetry between channels on the decision as to which input arrived first. This results in the amplitication of an intensity asymmetry between the channels in the form of latency differences between the signals as they arrive at more central decision functions. Thus, the effect of intensity asymmetry is increased as the signal passes through the channel. Consistent with this view is the fact that the reported time-intensity trade for "centering" of transients is around 50-65 $\mu \mathrm{sec} / \mathrm{dB}$ (Babkoff, Sutton, \& Barris, 1973; Deatherage \& Hirsh, 1959), while the effect of intensity asymmetry on temporal order judgment is reported as one or two orders of magnitude greater (Hirsh, 1959; Sanford, 1971). An additional piece of evidence can be added which bridges the gap between the "centering" experiment involving the perception and lateralization of one sound image ( $\Delta \mathrm{t} \leqslant 500 \mu \mathrm{sec})$, on the one hand, and the temporal order experiment $(\Delta t \geqslant 20 \mathrm{msec})$, on the other. This concerns the temporal separation of around $2-4 \mathrm{msec}$, i.e., the threshold for the breakup of the fused image into two dichotic stimuli.

In an earlier paper (Babkoff \& Sutton, 1966), we measured the effect of interaural intensity asymmetry $(\Delta \mathrm{I})$ on the $\Delta t$ necessary for the breakup of the "fused" image into two separately perceived stimuli at the two ears. This turned out to be approximately $175 \mu \mathrm{sec} / \mathrm{dB}$ at 28 to $31 \mathrm{~dB} \mathrm{SL}$. The effect of intensity asymmetry on the 2-4-msec segment of the dichotic temporal separation continuum lies between the time-intensity trade value reported for centering and that reported for temporal order judgments. This is consistent with the Sternberg and Knoll model, and helps to brige the gap between lateralization and temporal order judgment phenomena along the dichotic $\Delta$ t continuum.

\section{Physiological Implications}

The results of animal ablation studies, as well as studies of brain-damaged humans, argue for the importance of intact auditory cortex in the fusion of dichotic stimuli and their subsequent breakup into two stimuli as well as in the perception of temporal order.

Masterton, Jane, and Diamond (1967) compared the roles of the superior olives and of the auditory cortex for sound localization in cats. Cats with complete section of the trapezoid body show a deficit in the ability to locate a sound source. In contrast to normal cats, whose discrimination threshold is around $50 \mu \mathrm{sec}$, these animals require dichotic asymmetries of $500 \mu \mathrm{sec}$ to discriminate the direction of a sound source. Cats with intact superior olives, but without auditory cortex, cannot discriminate between sound sources even $180^{\circ}$ apart, despite the many monaural and binaural cues available to them, even if allowed to walk around in front of the sound source. Decorticate cats cannot transfer a discrimination between a single left and a single right signal to a dichotic pair of stimuli with left leading right vs. another pair of dichotic stimuli with right leading left, regardless of the dichotic temporal separation. This transfer can be performed by normal cats with ease. Masterton, Jane, and Diamond argue, therefore, that the role of the superior olive and lower brainstem structures seem to be the analysis of binaural disparity, whereas the role of the cortex must be to integrate sounds into a single auditory space. This argues that fusion of dichotic sounds and the subsequent separation of them into two sounds as $\Delta t$ exceeds some value, is a role performed by the cortex.

Lackner and Teuber (1973) have shown that patients with penetrating wounds of the left posterior cerebral hemisphere have abnormal fusion thresholds, that is, these patients still report hearing only a single "fused" sound. even though the interaural temporal asymmetry is sufficient for normal subjects to report hearing two sounds.

With regard to the perception of temporal order, Sw isher and Hirsh (1972) found that patients with left hemisphere lesions show a definite impairment in the judgment of the temporal order of dichotically 
presented transient stimuli.

As noted above, Sternberg and Knoll argue that the smaller effect of $\Delta I$ on binaural lateralization than on temporal order is consistent with the view that the decision function involved in binaural lateralization is closer to the periphery than the decision function involved in the judgment of temporal order. If we integrate the anatomic data into the independent channels model, then this view must be modified somewhat, at least with respect to the decision mechanism associated with the integration of dichotic sounds into one "fused" sound which appears to be a cortical mechanism. Perhaps there are several decision mechanisms, weighing arrival-time differences, interspersed in the auditory channels, such that the decision function responsible for the perceived location of a sound source and the discrimination of small interaural temporal differences is located early in the system, followed by the decision function responsible for "fusion" of the sound source which is cortical, followed eventually by the central temporal order judgment decision mechanism, also cortical, which receives inputs from auditory as well as other sensory systems. If the experimental paradigm introduced in this study allows a test of the Sternberg and Knoll model, then a specific prediction can be made concerning the diverse effect of interaural intensity asymmetry on the two limbs of the function shown in Figure 2. The model predicts that intensity asymmetry effects a decision function located earlier in the system less than it effects a decision function located at a later, more central level. Accordingly, the relative effect of $\Delta \mathrm{I}$ on the left descending limb of the function, illustrated in Figure 2, should be less than on the right ascending limb. This follows since, presumably, the breakup of a "fused" image and the subsequent decay of the effect of the lead on the lag stimulus occur at an earlier decision function than the judgment of temporal order. We are presently planning experiments such as this and others within the framework of the model suggested by Sternberg and Knoll.

\section{REFERENCES}

Babkoff, H., \& Sutron, S. Perception of temporal order and loudness judgments for dichotic clicks. Journal of the Acoustical Society of America, 1963, 35, 574-577.

Babkoff, H.. \& Sutron, S. End points of lateralization for dichotic clicks. Journal of the Acoustical Society of America, 1966, 39, 87-102.

Babkoff, H., \& Sutton, S. Monaural temporal interactions. Journal of the Acoustical Society of A merica, 1971, 50, 459-465.

Babkoff, H., Surton, S., \& Barris, M. Binaural interaction of transients: Interaural time and intensity asymmetry. Journal of the Acoustical Society of America, 1973, 53, 1028-1036.

BÉKÉsY. G. voN. Experiments in hearing. New York: McGraw'-Hill, 1960.

Carmon, A., \& Nachshon, I. Effect of unilateral brain damage on perception of temporal order. Cortex, 1971, 7, 410-418.
David, E. E., JR., Guttman, N., \& van Bergeiju, W. A. Binaural interaction of high-frequency complex stimuli. Journal of the Acoustical Society of A merica, 1959, 31, 774-782.

Deatherage, B. H., \& Hirsh, I. J. Auditory localization of clicks. Journal of the Acoustical Society of America, 1959. 31. $486-492$.

Efron, R. Temporal perception, aphasia and déja vu. Brain, 1963, 86, 403-424.

EFRON, R. Conservation of temporal information by perceptual systems. Perception \& Psychophysics, 1973, 14, 518-530.

Finney, D. J. Probit analysis. Cambridge University press, 1947.

Green, D. M., \& Henning, G. B. Audition. Annual Review of Psychology, 1969, 20, 105-128.

HarRIs, G. G. Binaural interactions of impulsive stimuli and pure tones. Joumal of the Acoustical Society of America, $1960,32$. 685-692.

HIRSH, I. J. Auditory perception of temporal order. Journal of the Acoustical Society of America, 1959, 31, 759-767.

Hirsh, I. J., \& Sherrick, C. E., JR. Perceived order in different sense modalities. Journal of Experimental Psychology, 1961, 62, 423-432.

Homick. J. L., Elfner, L. F., \& Bothe, G. G. Auditory temporal masking and the perception of order. Journal of the Acoustical Society of America, 1969, 45, 712-718.

Kietzman, M. L., \& Sutron, S. The interpretation of two-pulse measures of temporal resolution in vision. Vision Research. $1968,8,287-302$.

LACKNER, J. R., \& TEUBER, H. L. Alterations in auditory fusion thresholds after cerebral injury in man. Neuropsychologia, 1973, 11. 409.414.

Masterton, B., Jane, J. J., \& Diamond, I. T. Role of brainstem auditory structures in sound localization. I. Trapezoid body, superior olive and lateral lemniscus. Journal of Neurophysiology, 1967, 30, 341-359.

Oatley, K., Robertson, A., \& Scanlan, P. M. Judging the order of visual stimuli. Quarterly Journal of Experimental Psychology, 1969, 21, 172-179.

Patterson, J. H., \& Green, D. M. Discrimination of transient signals having identical energy spectra. Joumal of the Acoustical Society of America, 1970, 48, 894-905.

Robinson, D. Visual discrimination of temporal order. Science, 1967, 156, 1263-1264.

Rosenzweic, M. R., \& Rosenblith, W. A. Some electrophysiological correlates of the perception of successive clicks. Journal of the Acoustical Society of A merica, 1950, 22, 878-880.

Rutschman, R. Perception of temporal order and relative visual latency. Science, 1966, 152, 1099-1101.

RutschmanN, R. Visual perception of temporal order. In $S$. Kornblum (Ed.), Attention and performance IV. New York: Academic Press, 1973.

Rutschmann, J., \& Link, R. Perception of temporal order of stimuli differing in sense mode and simple reaction time. Perceptual Motor Skills, 1964, 18, 345-352.

SANFORD, A. J. Effects of changes in the intensity of white noise on simultaneity judgments and simple reaction time. Quarterly Journal of Experimental Psychology, 1971, 23, 196-303.

Sternberg, S., \& KNoll, R. L. The perception of temporal order: Fundamental issues and a general model. In S. Kornblum (Ed.), Attention and performance IV. New York: Academic Press, 1973.

Swisher, L., \& Hirsh, I. J. Brain damage and the ordering of two temporally successive stimuli. Neuropsychologia, 1972, 10 , 137-152.

ThOR, D. H. Dichoptic viewing and temporal discrimination: An attempted replication. Science, 1967, 158, 1704-1705.

Trimble, O. C. The theory of sound localization: A re-statement. Psychological Review, 1928, 35, 515-523.

(Received for publication August 15, 1974; revision accepted June 26,1975 .) 